ఠ

\title{
Application of surgical navigation in styloidectomy for treating Eagle's syndrome
}

\author{
This article was published in the following Dove Press journal: \\ Therapeutics and Clinical Risk Management \\ 12 April 2016 \\ Number of times this article has been viewed
}

\author{
Geng Dou' \\ Yu Zhang' \\ Chunlin Zong ${ }^{2}$ \\ Yuanli Chen ${ }^{2}$ \\ Yuxuan Guo ${ }^{2}$ \\ Lei Tian ${ }^{2}$ \\ 'The First Brigade of Students, \\ ${ }^{2}$ State Key Laboratory of Military \\ Stomatology, National Clinical \\ Research Center for Oral Diseases, \\ Shaanxi Key Laboratory of Oral \\ Diseases, Department of Cranio-facial \\ Trauma and Orthognathic Surgery, \\ School of Stomatology, The Fourth \\ Military Medical University, Xi'an, \\ People's Republic of China
}

Purpose: The present study aimed to evaluate the feasibility, accuracy, and clinical effect of intraoperative navigation for resection of elongated styloid process (ESP) in Eagle's syndrome. Patients and methods: Twelve patients with Eagle's syndrome with clinically and radiologically established diagnoses of ESP were included in this study. Preoperatively, all patients accepted three-dimensional computed tomography scan, and their skulls' digital imaging and communications in medicine data were inputed into the navigation system workstation to make a virtual surgical plan in advance. During surgery, the intraoperative navigation was performed to excise the ESP accurately for both intraoral (without tonsillectomy) and extraoral approaches following the virtual plan. Postoperatively, the amount of bleeding, duration of operation and hospitalization, and the length of resected styloid process (SP) were measured and compared with those cases that had traditional styloidectomy without the help of surgical navigation (SN). A simple visual analog scale questionnaire was also used to assess patients' satisfaction and the surgery effect after 3 months. Results: In total, 17 SPs from 12 patients were precisely resected by intraoral parapharyngeal approach and small cervical approach with the aid of SN. No severe complications occurred in any patients. The length of resected SPs was $21.93 \pm 14.26 \mathrm{~mm}$. The average amount of bleeding and duration of operation were $22.50 \pm 8.54 \mathrm{~mL}$ and $40.35 \pm 11.81$ minutes, respectively, which were all less than with traditional styloidectomy. The visual analog scale analysis showed that the discomfort in all patients was relieved, while ten patients' symptoms were improved greatly, and two patients had some improvement.

Conclusion: The higher accuracy of surgery, lesser amount of bleeding, decreased duration of surgery and hospitalization, absence of complications, and improved subjective symptoms indicated that $\mathrm{SN}$ is an effective and minimally invasive surgical procedure suitable for resection of ESP for treating Eagle's syndrome.

Keywords: elongation of styloid process, intraoperative navigation, oral and maxillofacial surgery, computer-aided surgery

\section{Introduction}

Elongated styloid process (ESP) syndrome, also known as Eagle's syndrome, is the term given to the symptomatic elongation of the styloid process (SP) or mineralization of the stylohyoid or stylomandibular ligament. It is named after American physician Eagle who first reported a series of uncomfortable symptoms, including throat pain and foreign body sensation on the affected side, reflex otalgia, head and neck pain, and hypersalivation, in 1937. ${ }^{1}$ The etiology of ESP has not been known clearly, and the aim of treatment was to relieve patients' complaints with conservative or surgical methods. Most physicians regard the resection of ESP as the preferred treatment at present. The surgical management of ESP consists of two major procedures: the transoral approach and the extraoral cervical approach. ${ }^{2,3}$ 
Although styloidectomy is not regarded as a sophisticated surgery, it is important to perform the surgical process precisely in consideration of its complicated surrounding anatomical structures and postoperative outcome. The extraoral or transcervical approach gives a more suitable anatomic exposure of both the SP and nearby structures, which would decrease the risk of vascular injury. Technically, the intraoral approach is relatively easy to perform due to less fascial dissection and less time consumption. However, it normally requires tonsillectomy and has disadvantages such as poor visualization, risk of neurovascular injury, and higher possibility of deep cervical infection.,

In the last 2 decades, with the application of computeraided surgery, craniomaxillofacial surgery has become more secure and accurate but less invasive. ${ }^{5}$ Surgical navigation (SN) is one of the most commonly used computer-aided surgery techniques with combination of medical imaging technology, computer technology, and stereotaxic technique. SN endows surgeons capabilities of doing surgery more precisely and less invasively in head and neck regions. ${ }^{6}$ It could help surgeons locate surgical instruments and target regions and surrounding structures without having to rely on subjective assessments and interpretations of image data sets, so that the individual surgery plan could be realized accurately. Besides, $\mathrm{SN}$ is also a great tool for teaching junior doctors and communicating with patients and other doctors. ${ }^{7}$ Because of these advantages, SN has been widely applied in the treatment of complex maxillofacial fractures, head and neck tumor resections, foreign body removals, and the reconstruction of craniomaxillofacial defects or deformities, and has improved not only the surgical accuracy and the success rate but also the treatment outcomes. ${ }^{6}$ However, there are currently no reports published about the application of SN in the styloidectomy for treating Eagle's syndrome. Our study was performed to investigate whether the intraoperative navigation system could be helpful for styloidectomy and evaluate its feasibility, accuracy, and clinical effects in treating Eagle's syndrome.

\section{Patients and methods}

The authors consulted the ethics committee in their hospital (Stomatology Hospital of The Fourth Military Medical University) and were advised this study did not need an individual ethics committee approval. Written informed consent was obtained from all of the patients.

\section{Clinical data}

Twelve patients who were suspected of having Eagle's syndrome from August 2012 to November 2014 were enrolled into this study. Their ages ranged from 37 years to 58 years, including four males and eight females. None of them had a previous history of tonsillectomy or histories of previous maxillofacial trauma. Their most common complaints were pharyngeal foreign body sensation, decreased neck mobility when turning to the affected side, restriction of mouth opening because of pain, discomfort of throat and tongue root, and odynophagia. Ipsilateral otalgia was also noted in three patients. They underwent detailed physical inspection, including palpation at the tonsillar fossa or the inside region of jaw angle on which a bony projection could be felt. To further establish the diagnosis, we followed the diagnostic criteria of Eagle's syndrome described by Prasad. ${ }^{8}$ We used a local anesthetic of $2 \%$ lidocaine to infiltrate at the site where the ESP was palpable and to see whether the patient's symptoms could subside temporarily. This test in each patient was positive and fulfilled the criteria for surgical intervention. Every patient was required to have an examination of threedimensional computed tomography (3D-CT) of skull (slice thickness $0.5 \mathrm{~mm}$ ). Radiology suggested that five patients had bilateral ESP and the other seven had unilateral ESP. The longest SP was $73.5 \mathrm{~mm}$, and the average length was $35.6 \pm 7.6 \mathrm{~mm}$. A visual analog scale (VAS) in questionnaire form was used to investigate patients' subjective characteristics and evaluate the level of patients' discomfort.

\section{Preoperative preparation}

Preoperatively, the skull's digital image data were input into navigation workstation (ENT/CMF Navigation Module; Brainlab, Feldkirchen, Germany). Through initial analysis (locate, measure, and mark the ESP), the surgeon could realize the location, shape, and direction of SP clearly. Then, a virtual operation plan was made in the workstation using iPlan software, and the preliminary excised position in SP was marked with different colors according to the length of SP and its relationship with adjacent structures. One principle for designing the resection spot was that the length of remnant SP should not be $>20 \mathrm{~mm}$, which is regarded as a normal length of SP in adults. ${ }^{9}$ The digital surgical plan was then transferred to SN machine in the theater.

\section{Intraoperative application and surgical technique}

Under general anesthesia, the patient was on the bed in the supine position. After local anesthetic was injected in the calvaria skin, a $5 \mathrm{~mm}$ incision was made to fix the dynamic reference frame in the skull with a screw. Registration process was completed by surface matching method with Z-touch and electromagnetic (EM) pointer so that the patient's dynamic 
3D-CT image could be seen on the screen of the navigation system. With the EM pointer's guide, we could detect the tip of SP according to the skull's image shown on the screen and locate it through the patient's cervical skin or parapharyngeal mucosa. Then, the incision was designed at the skin or mucosa that was most close to the tip of SP so that the surgeon could reach it with minimal injury. For the patients with intraoral approach, a 1.5-2 cm parapharyngeal incision was made in the mucosa superolateral to pharyngopalatine arch and superior to tonsillar bed, without tonsillectomy. For those with extraoral approach, a 2-3 cm incision was made at the anterior margin of the sternocleidomastoid muscle and posterior to the mandible angle.

After cutting the skin or mucosa, hemostatic forceps were used to do blunt dissection carefully, and the tip of SP was easily exposed by the assistance of EM pointer, which could help indicate the relationship between the pointer, SP, and surrounding structures on the screen. Then, a curved artery forcep was used to dissect, separate, and retract the muscles and ligaments attached with SP. The periosteum was stripped off from the tip to the base, which allowed visualization of the SP as much as possible. Then the EM pointer was inserted into the wound and moved along with the bony SP till it reached the planned resection spot, which could be seen by surgeons on the screen. After the resection spot was confirmed, the slim SP could be pinched off by a needle holder and the thick one could be cut down by a small round bur. The resected SP was then taken out, and drainage was set before wound closing if necessary. The patient with bilateral ESP underwent bilateral styloidectomy in the same surgery.

\section{Postoperative evaluation and statistical analysis}

For each patient, the amount of surgical bleeding, the duration of operation and hospitalization, and the length of the excised SP were recorded carefully. A VAS questionnaire was given to each patient before and 3 months after surgery to evaluate operation effect. Another 12 patients who were diagnosed ESP and received the traditional styloidectomy without SN assistance from 2008 to 2011 were chosen to serve as controls. Through reviewing their medical notes, the duration of operation and hospitalization and the amount of bleeding were acquired and compared with SN cases. Quantitative data in this study were expressed as mean $\pm \mathrm{SD}$, and significance was determined by Student's $t$-test with SPSS 19.0 (IBM Corporation, Armonk, NY, USA). Statistical significance was set at $P<0.05$.

\section{Results}

In total, 17 SPs from 12 patients were accurately resected and assisted with SN either by intraoral approach or by extraoral approach. No severe complications were noted in patients. The length of excised SPs was $21.93 \pm 14.26 \mathrm{~mm}$. The average amount of bleeding was $22.50 \pm 8.54 \mathrm{~mL}$. The durations of operation and hospitalization were $40.35 \pm 11.81$ minutes and 2.06 \pm 0.71 days, respectively. Comparing with control cases, all these indexes of SN cases were distinctly better (Table 1). Postoperatively, questionnaire survey showed that ten patients' symptoms were greatly improved, while two patients' discomfort was not entirely relieved. The postoperative VAS score was $1.83 \pm 0.64$, which was significantly lower than the preoperative score of 5.22 \pm 1.76 (Table 2).

\section{Clinical cases Case I}

A 56-year-old man came to our unit complaining of persistent and aggravated throat pain, and foreign body sensation in the right side over 3 years. He also experienced vague dull pains around the right lower face, although he had no face and head trauma history. He had been diagnosed for temporomandibular joint disorders and accepted medication therapy but had experienced little improvement. On physical examination, a hard strip body could be palpated in the top of right tonsillar fossa and his symptoms could be relieved by local injection of lidocaine. 3D-CT scan revealed that he had an SP of $40.8 \mathrm{~mm}$ in the right side, extending inferiorly toward the angle of the mandible. On the basis of these clinical and radiographic findings, Eagle's syndrome was diagnosed. After a deep discussion with us, the patient decided to have the right SP resected to relieve his symptoms. His 3D-CT data were input into the navigation workstation,

Table I Comparison of relevant indexes between navigation-assisted operation and the usual operation $(\bar{x} \pm S D)$

\begin{tabular}{llll}
\hline & $\begin{array}{l}\text { Average amount } \\
\text { of bleeding }(\mathbf{m L})\end{array}$ & $\begin{array}{l}\text { Duration of operation } \\
\text { (minutes) }\end{array}$ & $\begin{array}{l}\text { Duration of } \\
\text { hospitalization (days) }\end{array}$ \\
\hline Navigation cases $(\mathrm{n}=12)$ & $22.50 \pm 8.54^{*}$ & $40.35 \pm 11.8 I^{*}$ & $2.06 \pm 0.71 *$ \\
Conventional cases $(\mathrm{n}=\mathrm{I})$ & $45.70 \pm 9.86$ & $76.60 \pm 23.43$ & $3.92 \pm 0.76$ \\
\hline
\end{tabular}

Note: $* P<0.01$.

Abbreviation: SP, styloid process. 
Table 2 VAS score before and after the navigation aided styloidectomy ( $\mathrm{n}=12, \bar{x} \pm \mathrm{SD})$

\begin{tabular}{lll}
\hline Preoperative VAS & Postoperative VAS & P-value \\
\hline $5.22 \pm 1.76$ & $1.83 \pm 0.64$ & 0.0082 \\
\hline
\end{tabular}

Abbreviation: VAS, visual analog scale.

and a surgical plan was made by iPlan, which indicated the SP location, shape, and resection spot (Figure 1). Under general anesthesia, a $1.5 \mathrm{~cm}$ parapharyngeal incision was chosen with help from the real-time navigation technique. The incision located in the mucosa superolateral to pharyngopalatine arch and superior to tonsillar bed, which was very close to the tip of SP (Figure 2). After mucosa was cut, the ligament and muscles around SP were stripped off either by blunt dissection with curved hemostatic forceps or by scissor cutting. Then the EM pointer was inserted into the wound and moved along with the bony SP to find the planned resection spot, which was shown on the screen (Figure 3). In this way, the important anatomy structures around SP were protected without massive dissection. Then the SP was pinched off by a Stellbrink bone rongeur at the planned spot. The resected SP was taken out of the mouth, and the wound was closed by resorbable sutures. The patient received intravenous cefalotin $1.0 \mathrm{~g}$ just before the beginning of the operation and every 8 hours at the hospital for a period of 24 hours and then an oral administration of amoxicillin $500 \mathrm{mg}$ every 8 hours for 3 days more as an outpatient. At 1 month postoperatively, the mucosa wound healed well, and the patient was pleased to report that his previous symptoms of pain and foreign body sensation had resolved. He was subsequently discharged.

\section{Case 2}

A 43-year-old woman was referred to our department for persistent serious throat discomfort, swallowing pain, and dysphasia. She also felt tight in the neck when turning her head to the left. Her discomforts were thought to be related to chronic pharyngitis and were managed by conservative therapy, but no obvious improvement was gained. We gave her detailed physical examinations, and her symptoms of pain had localized to the left submandibular region. Then, a differential diagnosis of Eagle's syndrome was made. 3D-CT scan was adopted to evaluate the status of her SP and found surprisingly a giant SP of length $70.7 \mathrm{~mm}$ (Figure 4). Therefore, the patient was encouraged to have her giant SP resected to relieve her symptoms. The virtual surgical plan was made in the method described earlier (Figure 4). Because the SP was too strong to be taken out of the mouth whilst maintaining its integrity, a $2.5 \mathrm{~cm}$ submandibular incision was adopted under the guide of the navigation system. After the SP was fully exposed, a fine round bur was used to cut this strong SP. To make sure the bur only worked on the SP and the adjacent anatomical structures were protected, we set a dynamic reference frame on the handle of the bur and matched it with the navigation machine so that the bur could be used as an EM pointer. In this way, we could simultaneously see the exact movement and position of the round bur on the screen, which minimized the risk of injury of other surrounding structures (Figure 5). Then, a $61 \mathrm{~mm}$
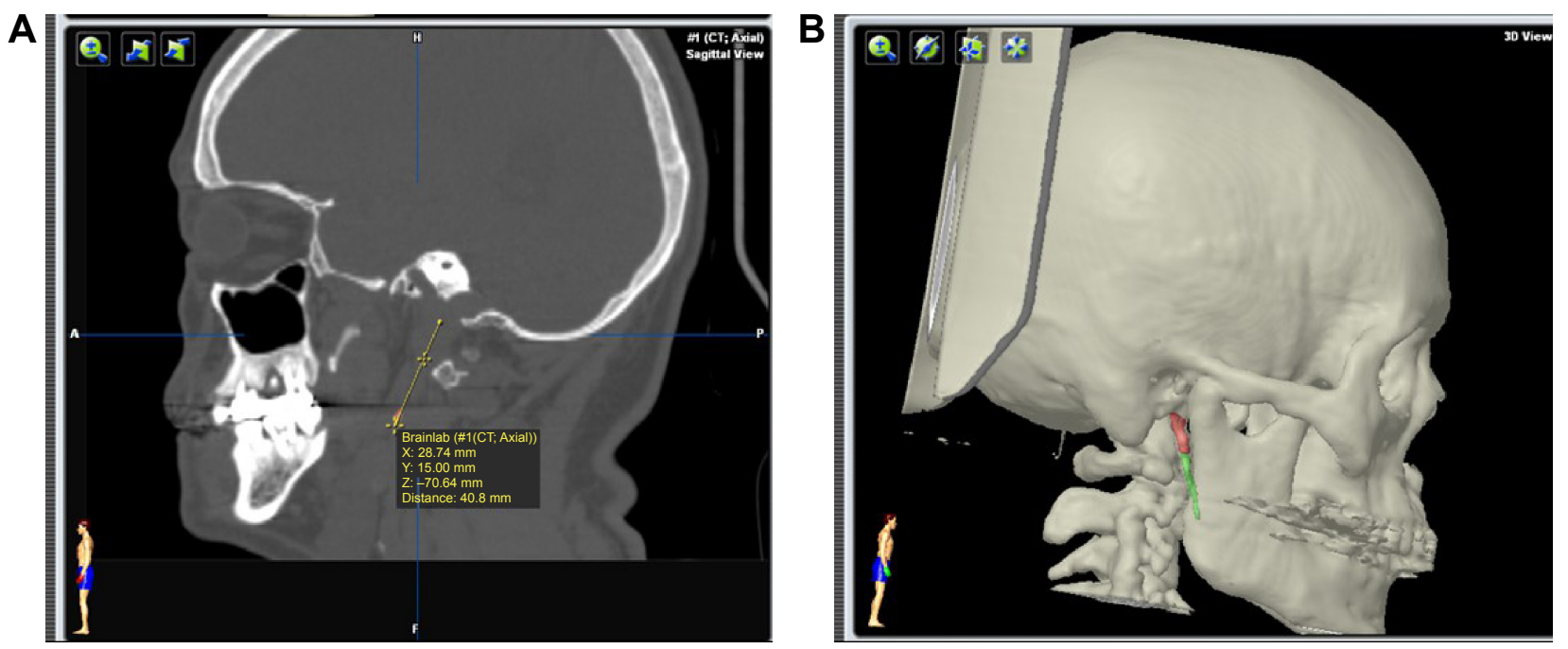

Figure I Preoperative analysis of the ESP in navigation workstation.

Notes: Patient's 3D-CT data were input into the navigation workstation to locate and measure the SP precisely, which showed its length was 40.8 mm (A). The virtual ESP was clearly seen using the software iPlan, and the resection spot was marked with green, while the residual was marked with red (B).

Abbreviations: 3D-CT, three-dimensional computed tomography; ESP, elongated styloid process; SP, styloid process. 

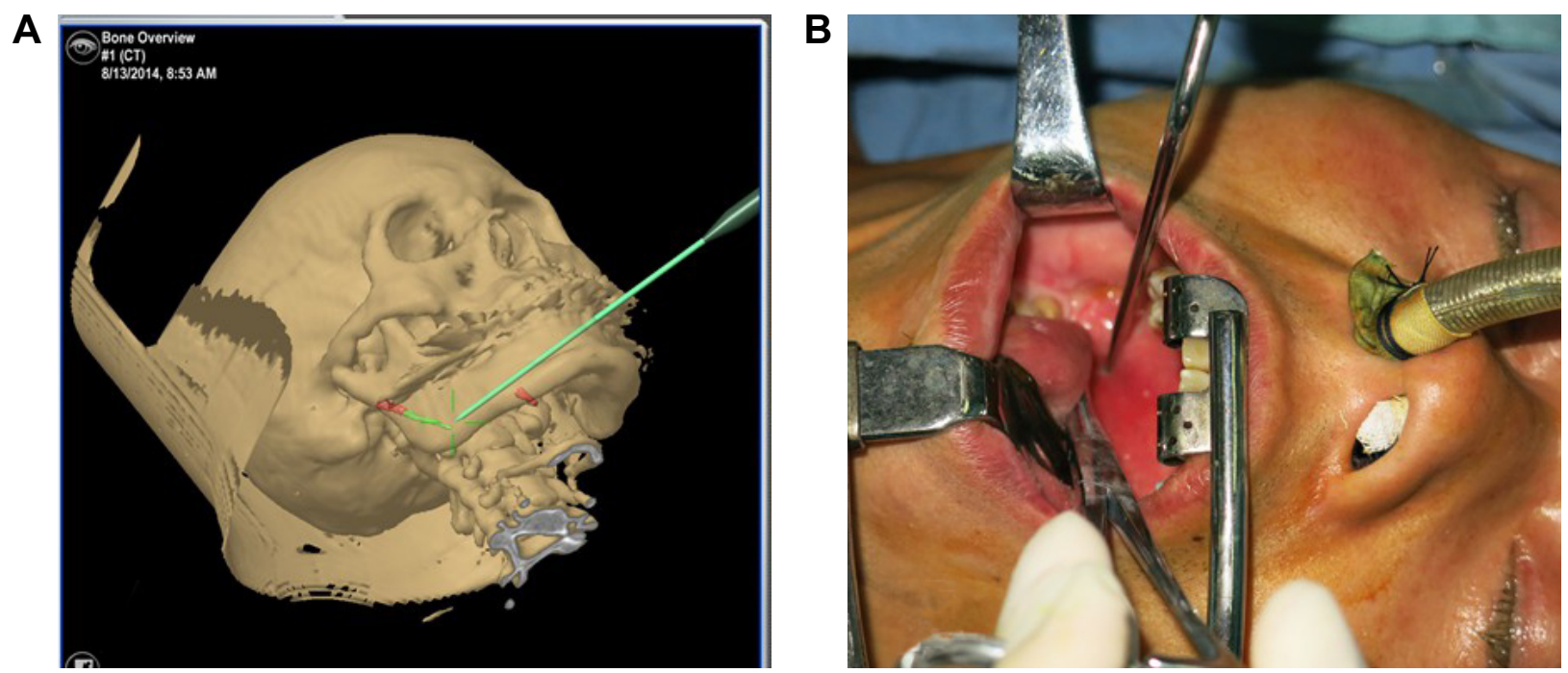

Figure 2 Intraoperative application of surgical navigation to orient the elongated SP.

Notes: After the registration process was completed, a pointer was used to find the tip of SP using the guide of the images shown in the navigation machine's screen (A). The green line denotes planned resection site in SP. Red color indicates the residual SP. Intraorally, the pointer showed the appropriate mucosa incision site that was closest to the SP (B).

Abbreviation: SP, styloid process.

section of the elongated right SP was excised according to the preoperative plan, in 43 minutes, with very little bleeding. The patient made an uneventful recovery without any complications. She was relieved of all her symptoms and subsequently discharged (Figure 6).

\section{Discussion}

Eagle's syndrome is a series of craniofacial and cervical discomfortable signs due to the elongation of SP or its abnormal structure. Its morbidity ranges from $1.4 \%$ to $30 \%$, most of which consists of middle-aged people and females. ${ }^{1,2}$ Because its nonspecific clinical symptoms can be confused with other disorders, the physicians need to make differential diagnosis with temporomandibular joint dysfunction, prosopalgia, sphenopalatine neuralgia, glossopharyngeal neuralgia, myofascial pain, mastoiditis, dentalgia, chronic amygdalitis, pharyngitis, and tumors. ${ }^{10}$ The clinical examination and the palpation are very helpful and specific for
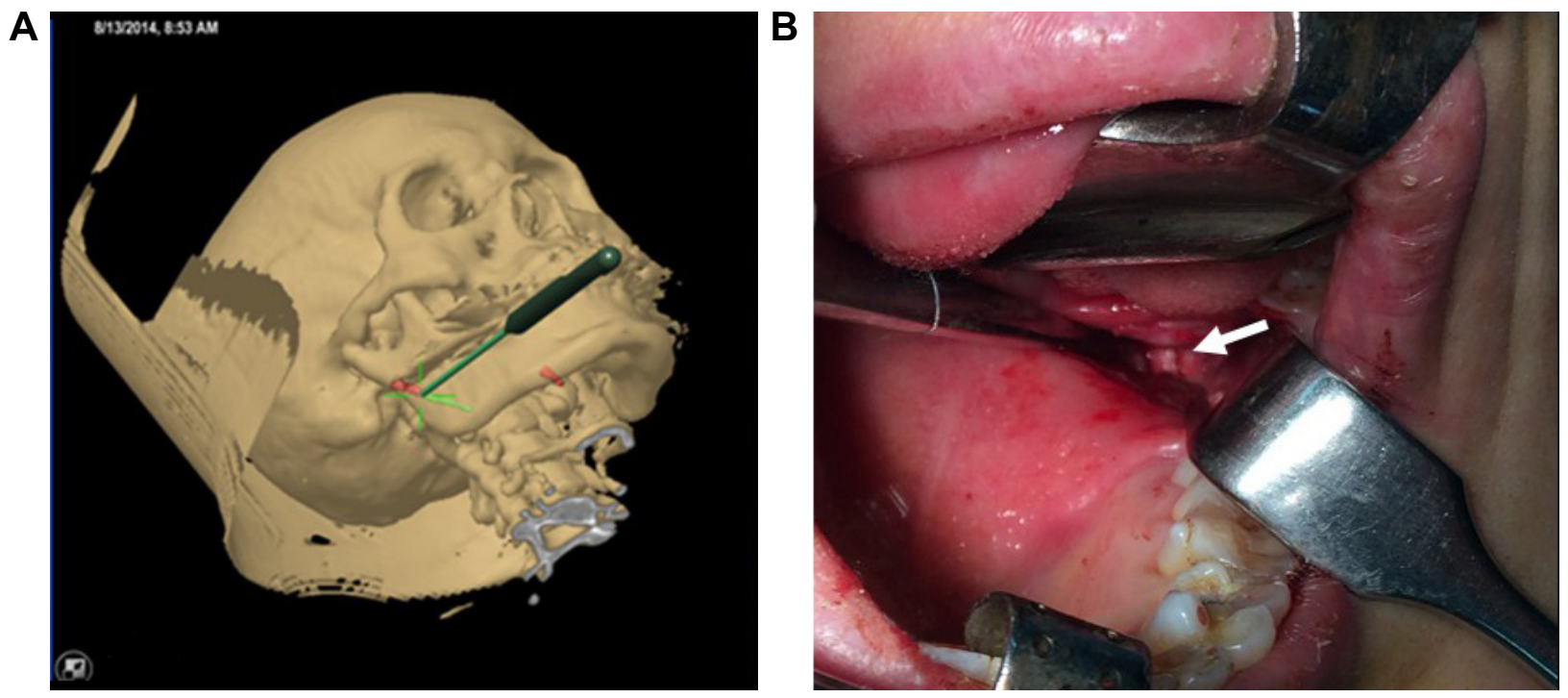

Figure 3 The intraoperative navigation was applied to expose the ESP by intraoral approach.

Notes: The pointer was inserted into the intraoral wound and the virtual resection site was found using the guide of the images shown in the navigation machine's screen (A). The green lines denote planned resection site in SP. Red color indicates the residual SP. The corresponding real resection spot was clearly exposed by the intraoral approach (B, white arrow).

Abbreviations: ESP, elongated styloid process; SP, styloid process. 
A

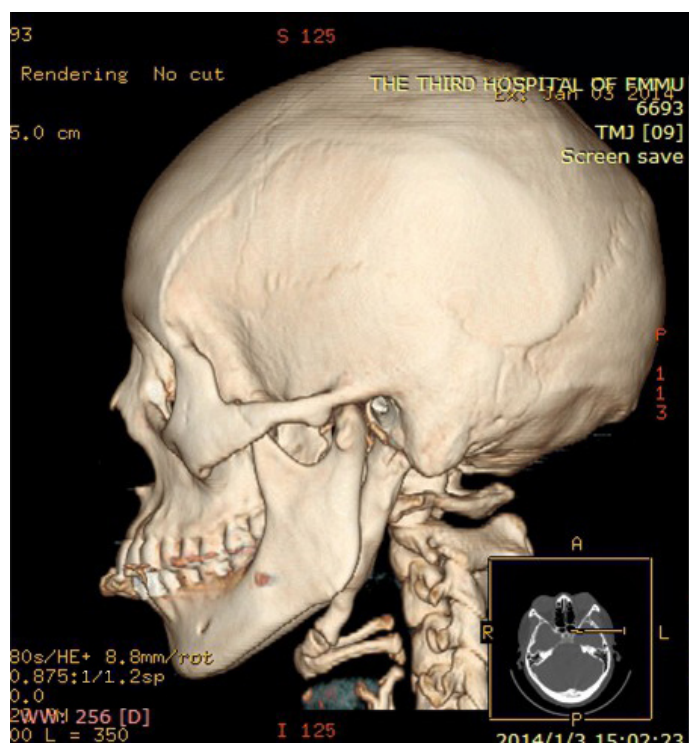

B

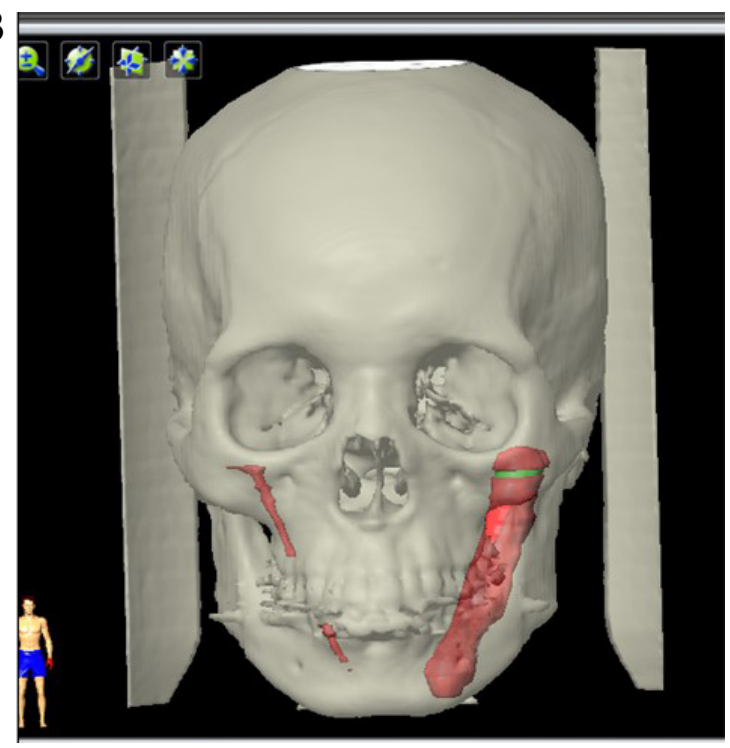

Figure 4 Images for preoperative analysis of the ESP and the virtual surgery plan.

Notes: 3D-CT showed that the patient had a giant styloid process in the left side, which explained her discomfort symptoms (A). A virtual surgery plan was made in the navigation workstation (B). The green line denotes planned resection site in the SP. Red color indicates the giant ESP.

Abbreviation: 3D-CT, three-dimensional computed tomography; ESP, elongated styloid process; SP, styloid process.
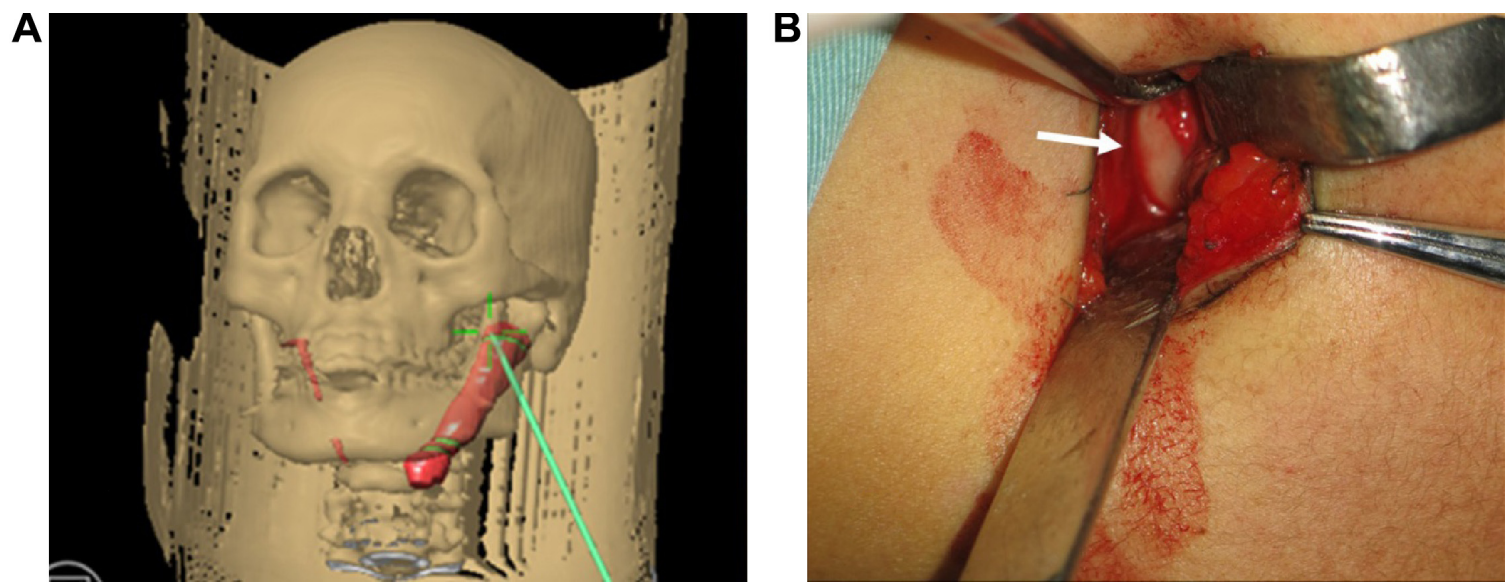

Figure 5 During the surgery, a fine round bur was matched with the navigation system so that it could be used as an EM pointer and its track could simultaneously be seen on the screen.

Notes: The bur had reached the planned resection site of SP (A). The green lines denote planned resection site in SP. Red color indicates the residual SP. The white arrow indicates the exposed styloid process by small cervical approach (B).

Abbreviations: EM, electromagnetic; SP, styloid process.
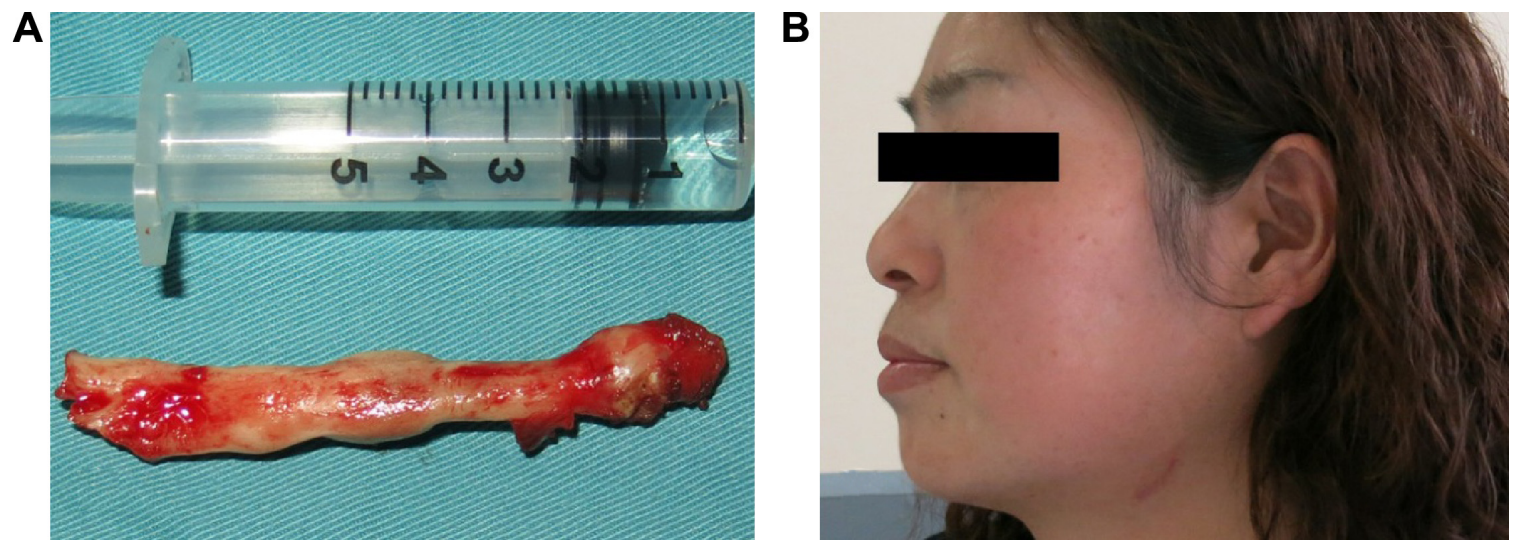

Figure 6 The resected giant elongated styloid process and the small cervical skin scar after extraoral approach.

Notes: The styloid process was excised in integrity $(\mathbf{A})$. The small cervical skin wound healed well after I month and the patient was satisfied with the cosmetic result (B). 
diagnosis. Besides, radiological examinations are very useful to gain an accurate diagnosis. 3D-CT can clearly visualize the structure of SP and its relationship with the adjacent anatomic structures directly and measure its length and angle precisely, which is very significant for the operation. So in our unit, each patient who is suspected of Eagle's syndrome is strongly recommended to have a 3D-CT scan.

At present, Eagle's syndrome can be treated either pharmacologically or surgically. For conservative approach, patients normally are treated with nonsteroidal anti-inflammatory medications or even carbamazepine, valporate, gabapentin, or amitriptyline to alleviate neuropathic sequelae. ${ }^{8}$ Lidocaine or steroid hormone is also often given to inject at the tip of SP through the tonsillar bed, which can relieve pain quite reliably. But the pharmaceutical therapy can only provide temporary relief and frequent injection is troublesome inevitably. Another reported nonsurgical therapy is to push the tip of SP hard outward and break it manually under local anesthesia. ${ }^{11}$ But due to the unsure effect and risk of injury to parapharyngeal tissues, it is not recommended.

The more reliable and permanent therapy of Eagle's syndrome is the surgical resection of the abnormal SP, which was first described by Eagle. ${ }^{1}$ At present, several transoral or external approaches to styloidectomy have been described, such as transcervical incision, preauricular incision, transoral incision through the fossa tonsillaris, retromolar incision, and paratonsillar incision. ${ }^{3,4,8}$ Each of them has some considerable advantages and disadvantages and should be performed individually on the basis of the patient's circumstances and the surgeon's experience. In general, the extraoral approach could provide adequate operation field to show the SP and its surrounding structures in the skull base, thus avoiding the unnecessary injury of neurovascular structures and the possibility of infections. Its major disadvantages include postoperative cosmetic deformity due to scar formation and requirement of extensive fascial dissection scope, which may result in longer duration of surgery and uncomfortable paresthesias of cutaneous nerves. ${ }^{3,8}$ Transoral approaches are relatively easy to perform and would not lead to any postoperative cosmetic deformities. Therefore, transoral approaches are considered to be the preferred option by most surgeons. ${ }^{3,12}$ However, the disadvantages of transoral approach include the possibility of deep cervical infection, the poor visualization, the difficulty of exposing and excising SP, and the risk of neurovascular injury. In classical transoral approach, ipsilateral tonsillectomy has to be performed in order to reveal the SP, which definitely increases surgical trauma and might lead to some sequelae. ${ }^{12,13}$ No matter which approach is adopted, the main concern is how to resect SP effectively in a minimally invasive way. Some novel methods have been developed to achieve this goal. Matsumoto et al described the benefit to identify small vessels and nerves and allow the surgeon to avoid injuries by using an endoscope at the beginning of the operation. ${ }^{14}$ But now, the surgery of SP still mainly depends on the surgeon's skill and experience.

With the combination of medical imaging technique, computer technique, and stereotaxic technique, the modern SN system can simulate operative procedures, make individual operation plans, and design optimal surgical access preoperatively. ${ }^{15}$ The real-time navigation also could improve the accuracy of the operation by guiding the surgeon's manipulation intraoperatively. After surgery, SN system could be used to evaluate the operation effect by merging the pre- and postoperative image data and comparing them by navigation software. Consequently, SN has demonstrated its tremendous superiority on craniofacial malformation correcting, the restoration of maxillofacial complex fracture, the reconstruction of maxillofacial bone defects, the resection of tumor, and the removal of foreign body. ${ }^{6}$ It sufficiently elucidates the modern surgery concept - "precise, effective, safe, minimally invasive and functional". ${ }^{16}$

Since the points of surgical approach for Eagle's syndrome are accurate identification and precise resection of SP to ensure the safety of surgery and good outcomes in a minimally invasive and cosmetic way, as far as possible, we believe SN could fully meet these requirements, considering what has been proved in other surgery fields. According to our study, SN has showed great advantages in enhancing surgery accuracy, shortening the surgical time, and reducing surgical trauma in the process of styloidectomy. The average amount of bleeding and the duration of operation and hospitalization in navigation cases were substantially less than the cases that underwent traditional styloidectomy. The greatly decreased postoperative VAS indicated that patients' subjective feeling significantly improved.

When SN is applied for ESP resection, it could provide benefits in three aspects as follows. Preoperatively, navigation system's workstation could analyze patients' digital imaging and communications in medicine data of skulls to acquire exact and visual detailed information about the specific location, morphological structure, direction, and diameter of the ESP. Then, the surgeons could design an optimal individual virtual surgical plan based on all this digital information. The second advantage of $\mathrm{SN}$ is that the surgeon could identify the spatial relationship between the SP and pharyngeal mucosa and design the optimal parapharyngeal incision that is closest to the tip of SP so that the surgeon could reach it directly without the need of tonsillectomy. Hence, the tonsilla and its 
immunologic function are preserved, and the surgical trauma is reduced. Even in the condition in which the extraoral approach has to be adopted, the SN will make it possible so that the styloidectomy could be done by a mini transcervical incision, which is normally not $>3 \mathrm{~cm}$ in length.

The third significant advantage of $\mathrm{SN}$ in surgical treatment of ESP is that the SN could provide a precise resection with minimal tissue dissection. In the conventional surgery, sometimes the elongated segment might not be fully excised because of the poor visualization and the complex surrounding tissues, unless the surgeon applies a massive dissection, which will result in increased surgical injury, longer recovery time, and poorer treatment experience to patients. With the help of SN, the surgeon could trace the surgical instruments' movement in time on the screen. It assists the surgeon to do styloidectomy, following the premade plan precisely, with less concern about the risk of neurovascular injury and with less fascial dissection. The decreased surgical duration and injury bring with them lower incidence of complications and better satisfaction of patients. The analysis of the follow-up data in our study has proved this.

Apart from these benefits, the surgeons should also pay some attention when using SN. First, the right cases should be chosen. Theoretically, SN is suitable for all patients with ESP who need to receive surgical treatment. But for those cases with less surgical difficulty, for example, the SP is easily palpated, fully calcified, and slim, the traditional approach could provide satisfactory exposure and resection, which could also reduce the medical cost and patient's financial burden. Second, guarantee the accuracy of SN. The inaccuracy normally comes from the following four factors: 1) inaccuracy of the 3D-CT data set and its processing and visualization; 2) inaccuracy of the navigation system used; 3) inaccuracy in registering the patient's head with the registration system; and 4) inaccuracy of the registration method used. ${ }^{17}$ In this study, we used surface matching to register patients with navigation system. According to some studies, the accuracy of surface matching is within $1.5 \mathrm{~mm} .{ }^{18}$ Although, it is not as accurate as invasive registration method, but it is good enough for styloidectomy. In the process of surface matching, there are two points that should be taken into account. The preoperative imaging data should involve all the skull as far as possible, to provide wide surface-matching region. The surgeon should further calibrate the registration accuracy by putting the EM pointer on the points of inner and outer canthus, nasion, and mesioincisal angle of the maxillary central incisor. If the image deviation exists, the surgeons should redo the registration process. Third, ensure the precise implementation of the preoperative plan. During surgery, the surgeons should ensure that surgical instruments' movement is guided by navigation all the way, to avoid any deviated surgical manipulation. The protection of adjacent neurovascular tissue should be focused on all the time, especially in the extraoral mini incision because of its poor surgical visualization. Last but not the least, trust the surgeon's experience and confidence. The surgeons should be aware that there always exist inevitable system errors and unpredictable machine obstacles in the navigation system. Surgeons should have their own judgment and strong confidence based on their clinical experience and surgical skill, rather than unconditionally depending on the navigation machine.

Our study proved that the navigation system was very helpful in the resection of ESP; however, there still exist two main limitations. In this study, all the styloidectomies aided by navigation were carried out by the author, while the traditional styloidectomy was completed by two other skillful surgeons. So it brought inaccuracy to some extent when we compared and analyzed the data from these two groups. A survey in greater scope or randomized controlled trial research will bring more accurate results. Since ESP resection is not a very complex surgery, the necessity of applying SN might be questionable. Navigation surgery is an advanced but expensive technology; patients have to spend more on it. But on the other hand, it will undoubtedly decrease the surgery duration, the length of hospital stay, and the risk of surgical complications, which would decrease medical cost. In our study, we did not compare the patients' cost in navigation cases against the traditional cases. A costeffectiveness analysis would be interesting since there is no reported economical evaluation for $\mathrm{SN}$ in craniomaxillofacial surgery. But some analysis in spinal surgery showed that $\mathrm{SN}$ technology is economically justified. ${ }^{19}$

Except for the two limitations of this study, there are some disadvantages from the SN system itself. Although the modern navigation system has a user-friendly program interface, the surgeons have to receive extra training before using this technique expertly. Using $\mathrm{SN}$ is meant to shorten the operating time in complex cases, however, SN may not reduce the total preoperative to postoperative duration, because the preoperative surgical planning and intraoperative registration processes are actually time consuming. Although SN has been applied in craniomaxillofacial surgery in many aspects, it is still not suitable for soft tissue and mandible surgery to date. ${ }^{20}$ But with the technical improvements, we believe that these shortcomings will be overcome and $\mathrm{SN}$ will be more friendly, convenient, and widely applied. 


\section{Conclusion}

The clinical study demonstrated that the application of SN in styloidectomy could result in a lesser amount of bleeding, decreased duration of surgery and hospitalization, absence of complications, and improved patients' subjective symptoms, and therefore, is an effective, precise, and minimally invasive method to treat Eagle's syndrome.

\section{Acknowledgments}

This work was supported by the Society Development Research Foundation of Shaanxi Province (2015SF140) and the National Natural Science Foundation of China (81202150).

\section{Disclosure}

The authors report no conflicts of interest in this work.

\section{References}

1. Werhun EL, Weidenhaft MC, Palacios E, Neitzschman H. Stylohyoid syndrome, also known as Eagle syndrome: an uncommon cause of facial pain. Ear Nose Throat J. 2014;93(9):384-385.

2. Becker C, Pfeiffer J. Eagle's syndrome. Am J Med. 2013;126(8): e3-e4.

3. Müderris T, Bercin S, Sevil E, Beton S, Kırıs M. Surgical management of elongated styloid process: intraoral or transcervical? Eur Arch Otorhinolaryngol. 2014;271(6):1709-1713.

4. Yavuz H, Caylakli F, Erkan AN, Ozluoglu LN. Modified intraoral approach for removal of an elongated styloid process. $J$ Otolaryngol Head Neck Surg. 2011;40(1):86-90.

5. Orentlicher G, Goldsmith D, Horowitz A. Applications of 3-dimensional virtual computerized tomography technology in oral and maxillofacial surgery: current therapy. J Oral Maxillofac Surg. 2010;68(8):1933-1959.

6. Austin RE, Antonyshyn OM. Current applications of 3-d intraoperative navigation in craniomaxillofacial surgery: a retrospective clinical review. Ann Plast Surg. 2012;69(3):271-278.
7. Saithna A, Dekker AP. The influence of computer navigation on trainee learning in hip resurfacing arthroplasty. Comput Aided Surg. 2009;14(4-6): $117-122$.

8. Prasad KC, Kamath MP, Reddy KJ, Raju K, Agarwal S. Elongated styloid process (Eagle's syndrome): a clinical study. J Oral Maxillofac Surg. 2002;60(2):171-175.

9. Prabhu LV, Kumar A, Nayak SR, et al. An unusually lengthy styloid process. Singapore Med J. 2007;48(2):e34-e36.

10. Ciorba A, Savini S, Morolli F, et al. Orofacial pain and Eagle syndrome: cues from a clinical series. Minerva Stomatol. 2014;63(10):361-367.

11. Kaur A, Singh A, Singal R, Gupta S. Is this way: self inflicted fracture of styloid process cures stylalgia. J Med Life. 2013;6(2):202-204.

12. Scheller K, Eckert AW, Scheller C. Transoral, retromolar, para-tonsillar approach to the styloid process in 6 patients with Eagle's syndrome. Med Oral Patol Oral Cir Bucal. 2014;19(1):e61-e66.

13. Fusco DJ, Asteraki S, Spetzler RF. Eagle's syndrome: embryology, anatomy, and clinical management. Acta Neurochir (Wien). 2012;154(7): 1119-1126.

14. Matsumoto F, Kase K, Kasai M, Komatsu H, Okizaki T, Ikeda K. Endoscopy-assisted transoral resection of the styloid process in Eagle's syndrome. Case report. Head Face Med. 2012;8:21-24.

15. Andrews BT, Thurston TE, Tanna N, et al. A multicenter experience with image-guided surgical navigation: broadening clinical indications in complex craniomaxillofacial surgery. J Craniofac Surg. 2015;26(4): 1136-1139.

16. Schulz C, Waldeck S, Mauer UM. Intraoperative image guidance in neurosurgery: development, current indications, and future trends. Radiol Res Pract. 2012;2012:1-9.

17. Schramm A, Gellrich N-C, Schmelzeisen R. Navigational Surgery of Facial Skeleton. Berlin; Heidelberg: Springer-Verlag; 2007:47-49.

18. Strong EB, Rafii A, Holhweg-Majert B, Fuller SC, Metzger MC. Comparison of 3 optical navigation systems for computer-aided maxillofacial surgery. Arch Otolaryngol Head Neck Surg. 2008;134(10): $1080-1084$.

19. Dea N, Fisher CG, Batke J, et al. Economic evaluation comparing intraoperative cone beam CT-based navigation and conventional fluoroscopy for the placement of spinal pedicle screws: a patient-level data cost-effectiveness analysis. Spine J. 2016;16(1):23-31.

20. Lübbers HT, Jacobsen C, Matthews F, Grätz KW, Kruse A, Obwegeser JA. Surgical navigation in craniomaxillofacial surgery: expensive toy or useful tool? A classification of different indications. J Oral Maxillofac Surg. 2011;69(1):300-308.
Therapeutics and Clinical Risk Management

\section{Publish your work in this journal}

Therapeutics and Clinical Risk Management is an international, peerreviewed journal of clinical therapeutics and risk management, focusing on concise rapid reporting of clinical studies in all therapeutic areas outcomes, safety, and programs for the effective, safe, and sustained use of medicines. This journal is indexed on PubMed Central, CAS,

\section{Dovepress}

EMBase, Scopus and the Elsevier Bibliographic databases. The manuscript management system is completely online and includes a very quick and fair peer-review system, which is all easy to use. Visit http://www.dovepress.com/testimonials.php to read real quotes from published authors. 